\title{
Natural alkaloid berberine activity against Pseudomonas aeruginosa MexXY- mediated aminoglycoside resistance: in silico and in vitro studies.
}

Emiliano Laudadio ${ }^{1}$, Nicholas Cedraro², Gianmarco Mangiaterra ${ }^{2}$, Barbara Citterio ${ }^{3}$, Giovanna Mobbili², Cristina Minnelli², Davide Bizzaro² ${ }^{2}$ Francesca Biavasco*2, Roberta Galeazzi*

${ }^{1}$ Dipartimento S.I.M.A.U., Università Politecnica delle Marche, via Brecce Bianche, 60131, Ancona, Italy; ${ }^{2}$ Dipartimento di Scienze della Vita e dell'Ambiente, Università Politecnica delle Marche, via Brecce Bianche, 60131, Ancona, Italy ${ }^{3}$ Dipartimento di Scienze Biomolecolari, sez. di Biotecnologie, Università degli Studi di Urbino "Carlo Bo", 61029 Urbino, Italy

TABLE S1. Structure and binding energy of top six compounds for site 1 and site 2 found from ensemble docking to MexY.

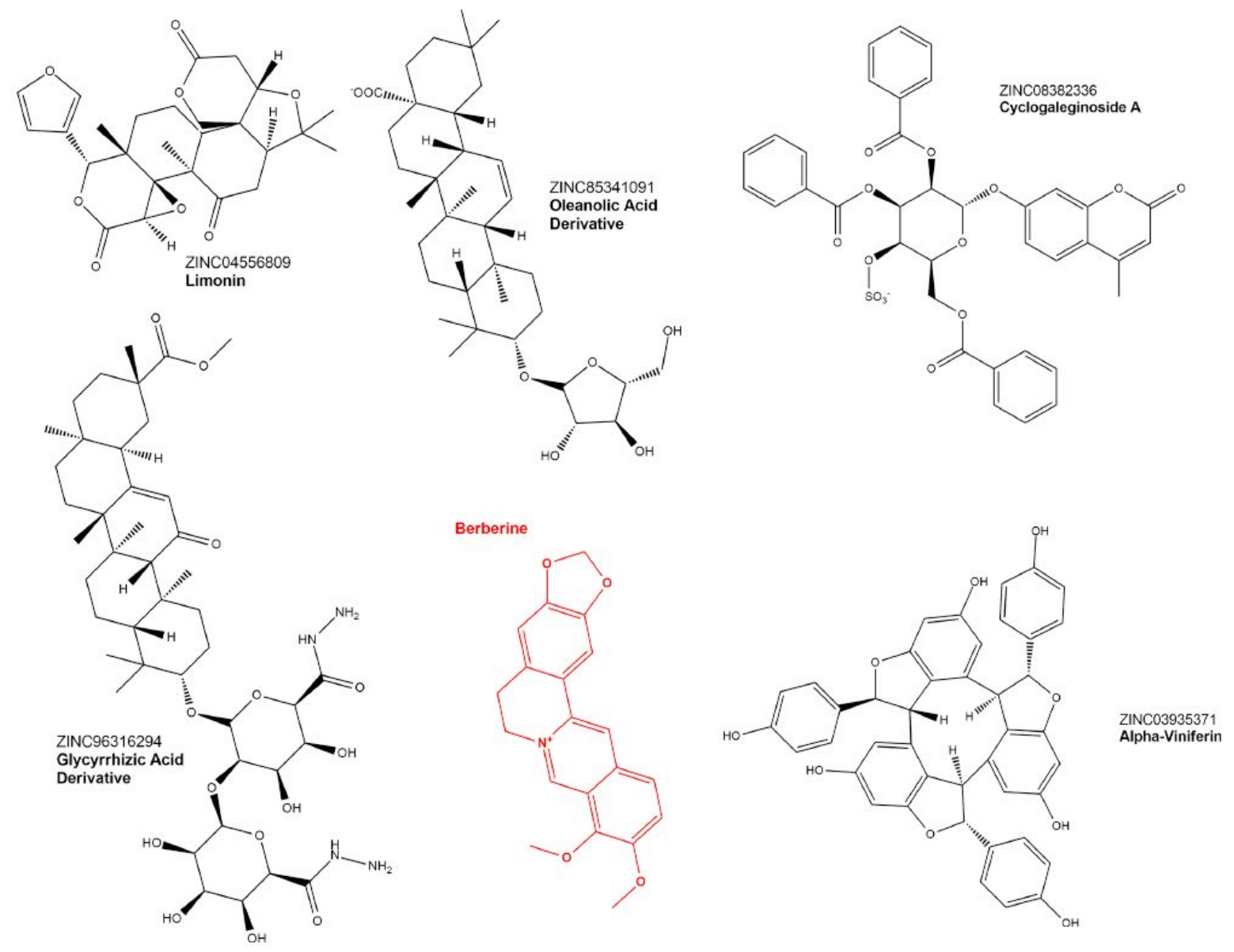




\begin{tabular}{|c|c|c|}
\hline Compound & $\begin{array}{c}\text { Binding } \\
\text { cleft }\end{array}$ & $\begin{array}{c}\text { Binding Energy } \\
\text { (Kcal/mol) }\end{array}$ \\
\hline $\begin{array}{c}\text { ZINC04556809 } \\
\text { Limonin }\end{array}$ & Site 2 & -11.44 \\
\hline $\begin{array}{c}\text { ZINC08382336 } \\
\text { Cyclogaleginoside A }\end{array}$ & Site 1 & -11.40 \\
\hline $\begin{array}{c}\text { ZINC96316294 } \\
\text { Glycyrrhizic Acid } \\
\text { Derivative }\end{array}$ & Site 1 & -12.10 \\
\hline $\begin{array}{c}\text { ZINC85341091 } \\
\text { Oleanolic Acid } \\
\text { Derivative }\end{array}$ & Site 2 & -11.33 \\
\hline $\begin{array}{c}\text { ZINC03935371 } \\
\text { alpha-Viniferin }\end{array}$ & Site 1 & -11.31 \\
\hline
\end{tabular}

List of Top 10 molecules found from ZINC database ensemble docking (results obtained in triplicate):

ZINC96316294 (see figure)

ZINC04556809 (see figure)

ZINC96316295

ZINC03935371 (see figure)

ZINC85340733

ZINC08382336 (see figure)

ZINC85341091 (see figure)

ZINC08382440

ZINC85341265

ZINC26187321

Table S2. Binding affinity and Site specificity for berberine and tobramycin

\begin{tabular}{|c|l|l|}
\hline Compound & Binding cleft & $\begin{array}{c}\text { Binding Energy } \\
\text { (Kcal/mol) }\end{array}$ \\
\hline berberine & Site 2 & -11.42 \\
\hline tobramycin & Site 2 & -8.21 \\
\hline
\end{tabular}
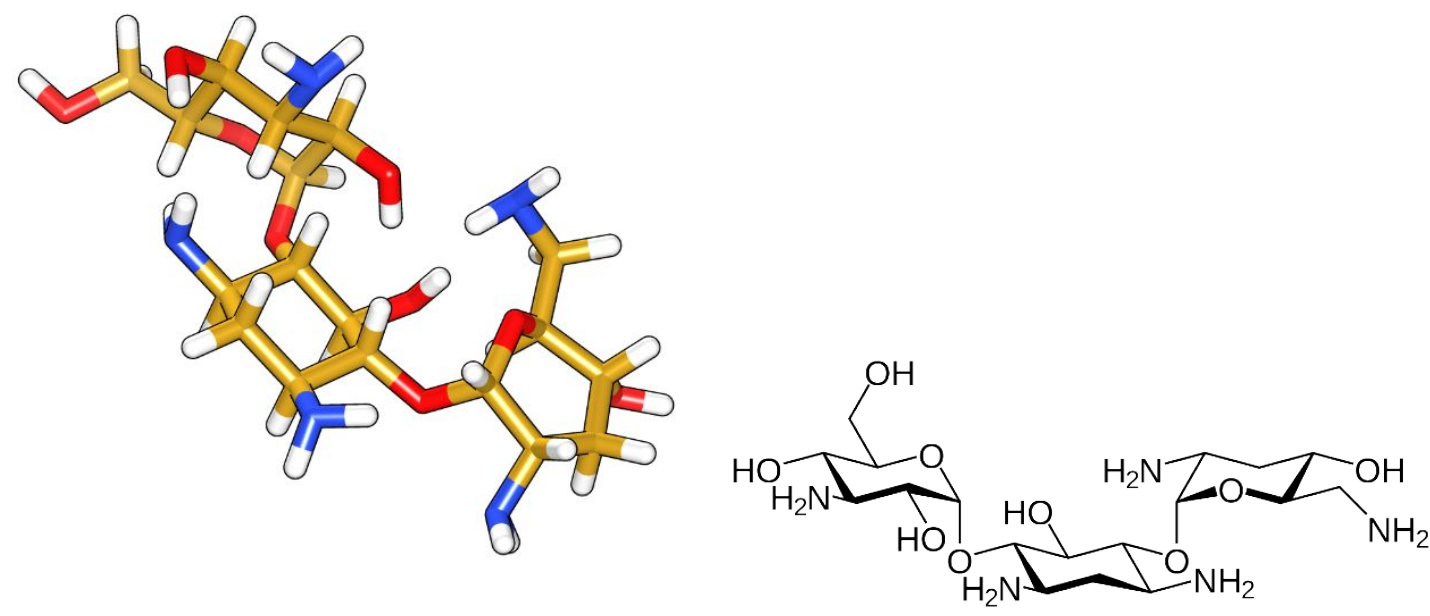

Figure S1. Tobramycin 3D and 2D structure

\section{Berberine-MexY and Tobramicyn-MexY molecular dynamics stabilization}

We evaluated the overall RMSD (Root Mean-Square Deviation) and the repositioning of the ligands inside their binding sites after stabilization, analyzing in details the interactions with the specific protein residues. The RMSD values obtained show that both systems are stabilized only after $10 \mathrm{~ns}$ of the whole MD simulation (Figure S2A). We did not extend our MD trajectories to far simulation 
times since we only want to focus on the ligands dynamical stabilization inside the binding clefts and to evaluate accurately their free energy of binding.

To this purpose, we carried out MM-PBSA calculation of the involved binding energies. In particular, we calculated the average values during all MD simulations, with snapshots generated every $10 \mathrm{ps,}$ and we plotted these values to better understand the transformations and stabilizations of the two models investigated (Figure S2B).
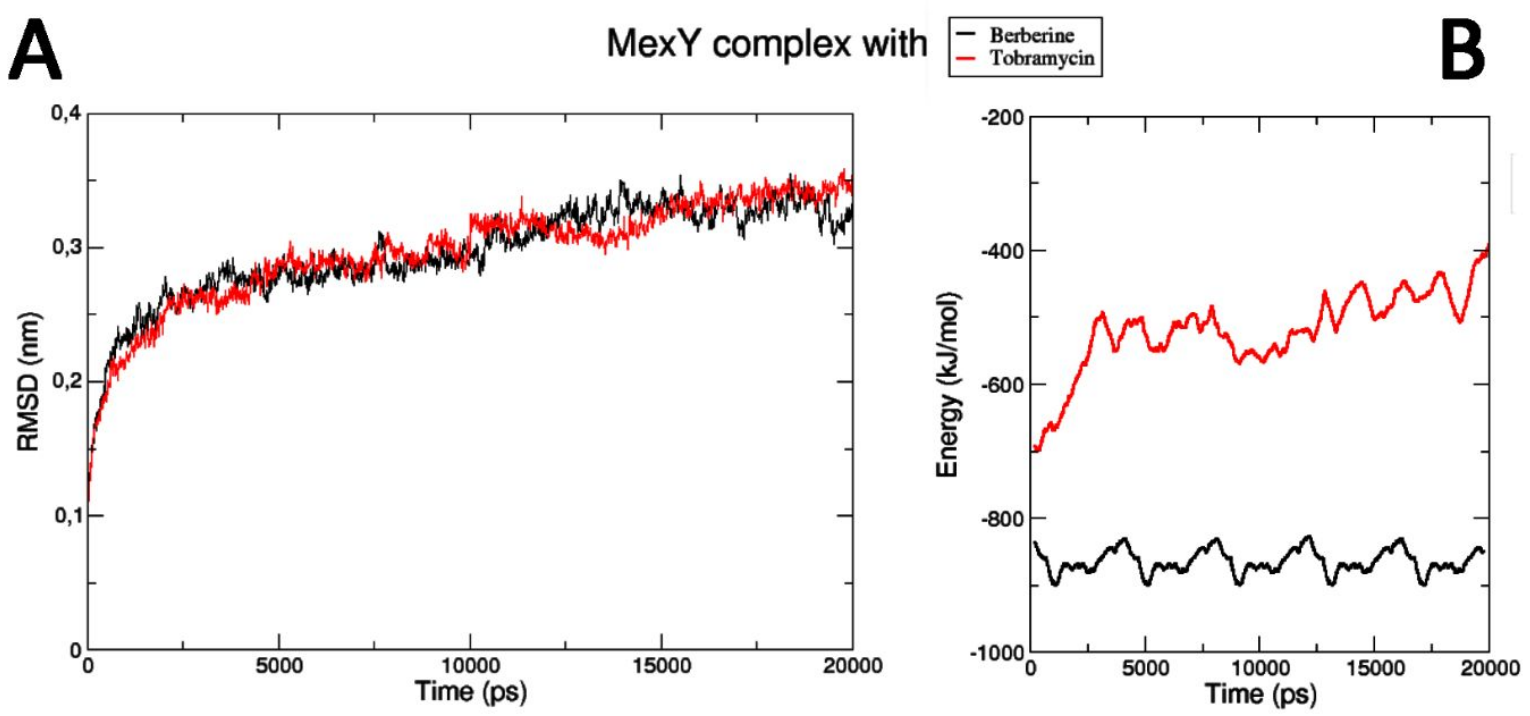

Figure S2. Root-Mean Square fluctuation (RMSD) values (A) and Total energy fluctuations (B) for the 20ns MD trajectory for the two simulated complexes.

Table S3. Checkerboard assays showing the synergistic effect of berberine on the tobramycin MIC against $P$. aeruginosa $\mathrm{C25}$

\begin{tabular}{|l|l|l|l|l|l|l|l|l|l|l|l|l|}
\hline & 1 & 2 & 3 & 4 & 5 & 6 & 7 & 8 & 9 & 10 & 11 & 12 \\
\hline $\mathrm{A}$ & $\mathrm{B}_{320}$ & $\mathrm{~B}_{320}$ & $\mathrm{~B}_{320}$ & $\mathrm{~B}_{320}$ & $\mathrm{~B}_{320}$ & $\mathrm{~B}_{320}$ & $\mathrm{~B}_{320}$ & $\mathrm{~B}_{320}$ & $\mathrm{~B}_{320}$ & $\mathrm{~B}_{320}$ & $\mathrm{~B}_{320}$ & $\mathrm{~B}_{320}$ \\
& $\mathrm{~T}_{0}$ & $\mathrm{~T}_{0.125}$ & $\mathrm{~T}_{0.25}$ & $\mathrm{~T}_{0.5}$ & $\mathrm{~T}_{1}$ & $\mathrm{~T}_{2}$ & $\mathrm{~T}_{4}$ & $\mathrm{~T}_{8}$ & $\mathrm{~T}_{16}$ & $\mathrm{~T}_{32}$ & $\mathrm{~T}_{64}$ & $\mathrm{~T}_{128}$ \\
\hline $\mathrm{B}$ & $\mathrm{B}_{160}$ & $\mathrm{~B}_{160}$ & $\mathrm{~B}_{160}$ & $\mathrm{~B}_{160}$ & $\mathrm{~B}_{160}$ & $\mathrm{~B}_{160}$ & $\mathrm{~B}_{160}$ & $\mathrm{~B}_{160}$ & $\mathrm{~B}_{160}$ & $\mathrm{~B}_{160}$ & $\mathrm{~B}_{160}$ & $\mathrm{~B}_{160}$ \\
& $\mathrm{~T}_{0}$ & $\mathrm{~T}_{0.125}$ & $\mathrm{~T}_{0.25}$ & $\mathrm{~T}_{0.5}$ & $\mathrm{~T}_{1}$ & $\mathrm{~T}_{2}$ & $\mathrm{~T}_{4}$ & $\mathrm{~T}_{8}$ & $\mathrm{~T}_{16}$ & $\mathrm{~T}_{32}$ & $\mathrm{~T}_{64}$ & $\mathrm{~T}_{128}$ \\
\hline $\mathrm{C}$ & $\mathrm{B}_{80}$ & $\mathrm{~B}_{80}$ & $\mathrm{~B}_{80}$ & $\mathrm{~B}_{80}$ & $\mathrm{~B}_{80}$ & $\mathrm{~B}_{80}$ & $\mathrm{~B}_{80}$ & $\mathrm{~B}_{80}$ & $\mathrm{~B}_{80}$ & $\mathrm{~B}_{80}$ & $\mathrm{~B}_{80}$ & $\mathrm{~B}_{80}$ \\
& $\mathrm{~T}_{0}$ & $\mathrm{~T}_{0.125}$ & $\mathrm{~T}_{0.25}$ & $\mathrm{~T}_{0.5}$ & $\mathrm{~T}_{1}$ & $\mathrm{~T}_{2}$ & $\mathrm{~T}_{4}$ & $\mathrm{~T}_{8}$ & $\mathrm{~T}_{16}$ & $\mathrm{~T}_{32}$ & $\mathrm{~T}_{64}$ & $\mathrm{~T}_{128}$ \\
\hline $\mathrm{D}$ & $\mathrm{B}_{40}$ & $\mathrm{~B}_{40}$ & $\mathrm{~B}_{40}$ & $\mathrm{~B}_{40}$ & $\mathrm{~B}_{40}$ & $\mathrm{~B}_{40}$ & $\mathrm{~B}_{40}$ & $\mathrm{~B}_{40}$ & $\mathrm{~B}_{40}$ & $\mathrm{~B}_{40}$ & $\mathrm{~B}_{40}$ & $\mathrm{~B}_{40}$ \\
& $\mathrm{~T}_{0}$ & $\mathrm{~T}_{0.125}$ & $\mathrm{~T}_{0.25}$ & $\mathrm{~T}_{0.5}$ & $\mathrm{~T}_{1}$ & $\mathrm{~T}_{2}$ & $\mathrm{~T}_{4}$ & $\mathrm{~T}_{8}$ & $\mathrm{~T}_{16}$ & $\mathrm{~T}_{32}$ & $\mathrm{~T}_{64}$ & $\mathrm{~T}_{128}$ \\
\hline $\mathrm{E}$ & $\mathrm{B}_{20}$ & $\mathrm{~B}_{20}$ & $\mathrm{~B}_{20}$ & $\mathrm{~B}_{20}$ & $\mathrm{~B}_{20}$ & $\mathrm{~B}_{20}$ & $\mathrm{~B}_{20}$ & $\mathrm{~B}_{20}$ & $\mathrm{~B}_{20}$ & $\mathrm{~B}_{20}$ & $\mathrm{~B}_{20}$ & $\mathrm{~B}_{20}$ \\
& $\mathrm{~T}_{0}$ & $\mathrm{~T}_{0.125}$ & $\mathrm{~T}_{0.25}$ & $\mathrm{~T}_{0.5}$ & $\mathrm{~T}_{1}$ & $\mathrm{~T}_{2}$ & $\mathrm{~T}_{4}$ & $\mathrm{~T}_{8}$ & $\mathrm{~T}_{16}$ & $\mathrm{~T}_{32}$ & $\mathrm{~T}_{64}$ & $\mathrm{~T}_{128}$ \\
\hline $\mathrm{F}$ & $\mathrm{B}_{10}$ & $\mathrm{~B}_{10}$ & $\mathrm{~B}_{10}$ & $\mathrm{~B}_{10}$ & $\mathrm{~B}_{10}$ & $\mathrm{~B}_{10}$ & $\mathrm{~B}_{10}$ & $\mathrm{~B}_{10}$ & $\mathrm{~B}_{10}$ & $\mathrm{~B}_{10}$ & $\mathrm{~B}_{10}$ & $\mathrm{~B}_{10}$ \\
& $\mathrm{~T}_{0}$ & $\mathrm{~T}_{0.125}$ & $\mathrm{~T}_{0.25}$ & $\mathrm{~T}_{0.5}$ & $\mathrm{~T}_{1}$ & $\mathrm{~T}_{2}$ & $\mathrm{~T}_{4}$ & $\mathrm{~T}_{8}$ & $\mathrm{~T}_{16}$ & $\mathrm{~T}_{32}$ & $\mathrm{~T}_{64}$ & $\mathrm{~T}_{128}$ \\
\hline
\end{tabular}




\begin{tabular}{|l|l|l|l|l|l|l|l|l|l|l|l|l|}
\hline $\mathrm{G}$ & $\mathrm{B}_{0^{*}}$ & $\mathrm{~B}_{0^{*}}$ & $\mathrm{~B}_{0^{*}}$ & $\mathrm{~B}_{0^{*}}$ & $\mathrm{~B}_{0^{*}}$ & $\mathrm{~B}_{0^{*}}$ & $\mathrm{~B}_{0^{*}}$ & $\mathrm{~B}_{0^{*}}$ & $\mathrm{~B}_{0^{*}}$ & $\mathrm{~B}_{0^{*}}$ & $\mathrm{~B}_{0^{*}}$ & $\mathrm{~B}_{0^{*}}$ \\
& $\mathrm{~T}_{0}$ & $\mathrm{~T}_{0.125}$ & $\mathrm{~T}_{0.25}$ & $\mathrm{~T}_{0.5}$ & $\mathrm{~T}_{1}$ & $\mathrm{~T}_{2}$ & $\mathrm{~T}_{4}$ & $\mathrm{~T}_{8}$ & $\mathrm{~T}_{16}$ & $\mathrm{~T}_{32}$ & $\mathrm{~T}_{64}$ & $\mathrm{~T}_{128}$ \\
\hline $\mathrm{H}$ & $\mathrm{B}_{0}$ & $\mathrm{~B}_{0}$ & $\mathrm{~B}_{0}$ & $\mathrm{~B}_{0}$ & $\mathrm{~B}_{0}$ & $\mathrm{~B}_{0}$ & $\mathrm{~B}_{0}$ & $\mathrm{~B}_{0}$ & $\mathrm{~B}_{0}$ & $\mathrm{~B}_{0}$ & $\mathrm{~B}_{0}$ & $\mathrm{~B}_{0}$ \\
& $\mathrm{~T}_{0}$ & $\mathrm{~T}_{0.125}$ & $\mathrm{~T}_{0.25}$ & $\mathrm{~T}_{0.5}$ & $\mathrm{~T}_{1}$ & $\mathrm{~T}_{2}$ & $\mathrm{~T}_{4}$ & $\mathrm{~T}_{8}$ & $\mathrm{~T}_{16}$ & $\mathrm{~T}_{32}$ & $\mathrm{~T}_{64}$ & $\mathrm{~T}_{128}$ \\
\hline
\end{tabular}

Different combinations of doubling concentrations of tobramycin (T), from 0.125 to $128 \mu \mathrm{g} / \mathrm{ml}$, and berberine (B), from 10 to $320 \mu \mathrm{g} / \mathrm{ml}$, were tested. Since berberine was dissolved in $50 \%$ DMSO and $50 \%$ methanol (final concentration 1\% DMSO 1\% methanol), the effect of the two solvents alone $\left(\mathrm{B}_{0^{*}}\right)$ was assessed as well. Bacterial growth is indicated in the table as a yellow box. 